\title{
Recherche, médecine et marché : la génétique du cancer du
} sein

In: Sciences sociales et santé. Volume 18, n4, 2000. pp. 29-51.

Citer ce document / Cite this document :

Cassier Maurice, Gaudillière Jean-Paul. Recherche, médecine et marché : la génétique du cancer du sein. In: Sciences sociales et santé. Volume $18, n^{\circ} 4,2000$. pp. 29-51.

doi : $10.3406 /$ sosan.2000.1504

http://www.persee.fr/web/revues/home/prescript/article/sosan_0294-0337_2000_num_18_4_1504 


\section{Résumé}

Résumé. La génétique du cancer du sein est marquée par une forte intégration de la science biomédicale et du marché. La recherche sur les gènes de prédisposition au cancer du sein s'est effectuée dans le cadre de nouveaux liens entre laboratoires universitaires, laboratoires cliniques et entreprises. Les savoirs sur les gènes sont appropriés par des brevets détenus principalement par une start-up américaine, Myriad Genetics. Le fait le plus nouveau concerne le lien entre la propriété intellectuelle et la construction d'un nouveau marché médical. Forte de ses brevets et son outil industriel, Myriad Genetics a développé un marché privé des tests génétiques qui s'autonomise vis-àvis du contexte médical. Notre article, fondé sur une comparaison France/États-Unis, analyse dans un premier temps différents modes de production de la recherche confrontés à la perspective du marché, puis dans un second temps oppose deux modes de fourniture et d'usage des tests génétiques, le modèle de la start-up et celui de la clinique.

\section{Resumen}

Ciencia, medicina y mercado: la genética del cancer del seno.

El desarrollo de la genética del cancer del seno hacia el fin de los años 80 ilustra la constitución de una esfera autónoma de los tests de prédisposición genética a ciertas formas de cancer que se instala en un contexto de intensificación de las relaciones entre investigación, medicina y mercado. El artículo clarifica esta dinámica a partir de una comparación entre Francia y los Estados Unidos. Para hacerlo estudia :

-la constitución de las bases de conocimientos correspondientes a estos trabajos ;

-las estrategias de apropiación desarrolladas por los investigadores, en particular el papel crucial de las patentes ;

-las relaciones entre genetistas y oncólogos, entre realización de los tests y utilización de sus resultados.

Opone un modelo « start-up » a un modelo " clínico » de emergencia de la medicina predictiva que podemos calificar respectivamente de eco- nomia « moral » y de economía « política » de los tests genéticos.

\section{Abstract}

Research, medicine and the market: breast cancer genetics

Breast cancer genetics is marked by a high level of integration of biomedical science and the market. Research on breast cancer predisposition genes is performed in the context of new partnerships between university laboratories, clinical laboratories and firms. Knowledge on genes is appropriated through patents held primarily by a US start-up, Myriad Genetics. The latest fact concerns the link between intellectual property and the building of a new medical market. Myriad Genetics, strengthened by its patents and its industrial apparatus, has developed a private genetic test market that is increasingly autonomous vis-à-vis the medical world. Our article, based on a comparison between France and the United States, analyses different modes of production of research faced with market prospects. It then compares two modes of provision and use of genetic tests, and the start-up model as opposed to the clinic model. 


\title{
Recherche, médecine et marché : la génétique du cancer du sein
}

\author{
Maurice Cassier*, Jean-Paul Gaudillière**
}

\begin{abstract}
Résumé. La génétique du cancer du sein est marquée par une forte intégration de la science biomédicale et du marché. La recherche sur les gènes de prédisposition au cancer du sein s'est effectuée dans le cadre de nouveaux liens entre laboratoires universitaires, laboratoires cliniques et entreprises. Les savoirs sur les gènes sont appropriés par des brevets détenus principalement par une start-up américaine, Myriad Genetics. Le fait le plus nouveau concerne le lien entre la propriété intellectuelle et la construction d'un nouveau marché médical. Forte de ses brevets et son outil industriel, Myriad Genetics a développé un marché privé des tests génétiques qui s'autonomise vis-à-vis du contexte médical. Notre article, fondé sur une comparaison France/États-Unis, analyse dans un premier temps différents modes de production de la recherche confrontés à la perspective du marché, puis dans un second temps oppose deux modes de fourniture et d'usage des tests génétiques, le modèle de la start-up et celui de la clinique.
\end{abstract}

Mots clés : appropriation des gènes, marché des tests génétiques, régulation des usages, modèle de la clinique, modèle de la start-up.

\footnotetext{
* Maurice Cassier, sociologue, CERMES, 182, boulevard de la Villette, 75019 Paris. ** Jean-Paul Gaudillière, historien, CERMES, 182, boulevard de la Villette, 75019 Paris.
} 
La société américaine Myriad Genetics, localisée à Salt Lake City, connue pour son implication dans les recherches sur les gènes de prédisposition au cancer du sein, présente son activité dans des termes sans ambiguité sur la nature commerciale de cette organisation : «Myriad Genetics Laboratories, which develops and markets proprietary genetic testing services and has introduced products in the fields of predictive medicine and personalized medicine ». Myriad Genetics est loin d'être une exception. Les recherches en génétique médicale et en génomique, associées depuis une vingtaine d'années à la promesse d'une nouvelle médecine (Cohen, 1993, Dausset, 1998), se sont très vite déroulées dans la perspective, sinon dans le contexte, de l'appropriation et des usages marchands des connaissances.

Initiés dès l'émergence des technologies de l'ADN et considérablement renforcés par la montée en puissance des programmes d'étude du génome humain, les rapprochements entre science biomédicale et marché opèrent en plusieurs points. Premièrement, de nombreuses sociétés privées de biotechnologie sont créées par des chercheurs à la périphérie des universités ou des organismes publics de recherche pour commercialiser leurs données. Un marché privé de la recherche génomique s'est ainsi constitué. Deuxièmement, de nombreux brevets sont pris sur les séquences de gènes et leurs applications diagnostiques ou thérapeutiques, par des firmes privées mais aussi par les institutions de recherche publique ou académique (les NIH - National Institutes of Health - ouvrirent la voie en déposant en 1991 une demande de brevet sur plusieurs milliers de séquences d'ADN complémentaire). Troisièmement, les sociétés de biotechnologie ne se contentent pas de vendre de l'information génétique. À l'instar de Myriad Genetics, elles construisent des laboratoires de tests qui sont désormais offerts dans un contexte commercial et passent des accords avec les compagnies d'assurance pour le remboursement de ces tests aux patients. Elles contribuent ainsi à la naissance de nouveaux services et à façonner les usages médicaux des tests génétiques.

Le domaine du cancer du sein est emblématique de cette nouvelle génétique humaine, des réseaux de recherche et d'innovation qui la supportent, et des relations qu'elle entretient avec les marchés de la recherche et de la santé. Les recherches sur les gènes de prédisposition au cancer du sein ont commencé au milieu des années quatre-vingt avec les premières étapes du programme génome initié par les NIH et le Département de l'énergie américain. Il s'agissait, pour les chercheurs en génétique qui jusqu'ici avaient travaillé sur des maladies peu répandues, de changer d'échelle et d'aborder "une pathologie qui ait de l'importance » (MC King, entretien). Deux gènes, dits $B R C A l$ et $B R C A 2$, dont les mutations augmentent considérablement le risque d'apparition d'un cancer du 
sein, furent localisés au début des années quatre-vingt-dix. On attendait de leur connaissance des applications thérapeutiques autant que diagnostiques. La rencontre entre science et marché a été très rapide puisque Myriad Genetics fut créée en 1991 par un chercheur de l'université de l'Utah. Myriad Genetics passa un accord avec la firme pharmaceutique Eli Lilly sur l'identification de $B R C A I$ au début des années quatre-vingt-dix. Des brevets sur les gènes $B R C A$ furent déposés en 1994 et 1995, par deux sociétés concurrentes, Myriad Genetics et OncorMed, par plusieurs universités et par une fondation caritative britannique, la Cancer Research Campaign. À partir de 1996, les tests de dépistage de mutations dans les séquences de $B R C A l$ et $B R C A 2$, jusque là réalisés dans un contexte de recherche, deviennent des enjeux commerciaux.

Ces développements semblent justifier la thèse selon laquelle la fin du siècle a vu l'émergence d'un autre mode de production des savoirs, plus proche des contextes d'application et de valorisation des connaissances (Gibbons et al., 1994). Ils suggèrent de plus que, dans le cas des biotechnologies, ces nouvelles façons de lier laboratoire, industrie et entreprise contribuent à modeler les cadres d'usages et les significations attribuées aux innovations médicales issues de la génomique, notamment en favorisant la constitution de nouveaux modes de gestion du risque génétique. Cet article entend éclairer les dynamiques de cet ensemble d'innovations en partant d'une comparaison des configurations contrastées qu'a prise, aux États-Unis et en France, la génétique du cancer du sein. La première section de l'article porte sur les modes de production et d'échange des savoirs dans la phase de recherche des gènes $B R C A$, la deuxième section aborde les cadres de fourniture et d'usage des tests génétiques (1).

(1) Nous avons réalisé des entretiens avec des généticiens, cancérologues et épidémiologistes français qui ont été associés à la recherche des gènes de prédisposition au cancer du sein et avec quelques-uns des chercheurs américains ayant joué un rôle important dans la découverte et l'exploitation des gènes BRCAI et BRCA2. Nous avons également recucillis des entretiens auprès de conscils en propriété industrielle, du responsable de la valorisation de l'Institut Curie, des responsables de l'Association nationale des praticiens de génétique moléculaire. Nous avons collecté les textes des brevets sur les gènes $B R C A$, les contrats européens qui supportèrent le Breast Linkage Consortium, les guidelines des associations de chercheurs et de praticiens aux ÉtatsUnis et en France. 


\section{La chasse aux gènes : les modes de production des savoirs}

La chasse aux gènes mobilisa quatre modes de production des savoirs que nous présenterons successivement :

- des échanges académiques bilatéraux entre cliniciens et chercheurs ;

- des réseaux de recherche collective : ateliers génome humain et consortium international sur le cancer du sein ;

- la création d'un secteur privé de la recherche : une start-up de génomique ;

- le développement d'équipes de recherche génétique dans le contexte de la clinique : les équipes françaises des centres anticancéreux soutenues par la Ligue contre le cancer.

Le premier mode de production des savoirs utilise des liens assez communs entre chercheurs académiques, à savoir des échanges de données régulés par des normes tacites de confiance et de réciprocité. Les premiers travaux en génétique du cancer du sein ont été initiés par des chercheurs universitaires qui mobilisaient les ressources disponibles dans les réseaux cliniques et académiques. À l'époque, les collections de données familiales sur les cancers étaient rares (jusqu'à la fin des années soixante-dix, la question de l'hérédité des cancers était scientifiquement marginale) (2). L'un des rares lieux où l'on avait pris pour objet de recherche le caractère héréditaire de nombreux cancers humains était la clinique de Henry Lynch à Omaha dans le Nebraska. Lynch est à la tête d'une consultation de génétique du cancer qui recrute dans toute la région et qui lui sert de source de matériel pour construire une collection de cas.

La collection de Lynch comporte des fiches individuelles de patients, des arbres généalogiques reconstitués après entretiens et les tissus prélevés sur certains patients. Elle comporte plusieurs milliers de pedigrees. Elle alimente dans un premier temps les publications de Lynch et de ses assistants (un ouvrage parait en 1974 sur les cancers héréditaires). La chasse aux gènes de prédisposition, à partir du début des années quatre-vingt lui confère un nouvel intérêt. Plusieurs chercheurs font le déplacement jusqu'à Omaha pour négocier un accès à cette collection. Celle-ci n'est pas mise dans le domaine public pas plus qu'elle n'est directement exploitée par Lynch qui ne dispose pas d'un laboratoire de géné-

(2) Des malades s'adressent parfois aux médecins pour les alerter sur la fréquence des cancers dans leur famille (certains leur fournissent des arbres généalogiques), mais les pratiques de recueil systématique sont peu fréquentes. Plus généralement, on peut identifier deux vagues d'intérêt pour l'hérédité du cancer : des annćes trente aux années cinquante, puis à partir des années quatre-vingt (Gaudillière, 1999). 
tique moléculaire. Après une courte collaboration avec une généticienne américaine, Lynch choisit de communiquer ses données sur le cancer du sein et de l'ovaire à un chercheur lyonnais, G. Lenoir.

Cette collaboration se fait sans accord écrit, sur la base d'une relation interpersonnelle fondée sur la confiance (pour Lynch, Lenoir est un chercheur de qualité qui saura valoriser sa collection), l'exclusivité (Lynch garantit à Lenoir un accès exclusif aux données sur le cancer du sein et de l'ovaire et écarte d'autres universitaires intéressés), et la réciprocité (Lynch bénéficie d'un retour sous forme de citations dans toutes les publications qui utilisent sa collection). Si bien que les échantillons et les pedigrees de Lynch se retrouvent dans le laboratoire de Lenoir au Centre de recherche international sur le cancer de Lyon et chez lui seul. Cette collaboration est durable (en 1999 Lynch est toujours signataire des articles de Lenoir).

D'une façon plus générale, au cours des années quatre-vingt et au début des années quatre-vingt-dix, les échanges se structurent sous forme de réseaux de recherche collaborative qui constituent un second mode de production des connaissances. Par exemple, M.-C. King, qui localisera le premier gène de prédisposition au cancer du sein, participe dans la seconde moitié des années quatre-vingt au réseau du Centre d'étude sur le polymorphisme humain (CEPH) mis en place par D. Cohen et J. Dausset en 1983. Le dispositif de recherche repose sur le test de tous les marqueurs potentiels sur un même pool de familles dont les arbres sont bien connus et l'ADN mis en commun (il s'agit des familles réunies par J. Dausset pour la mise en évidence des gènes contrôlant la compatibilité tissulaire et le rejet des transplantations dans les workshops HLA). Les résultats des études de localisation des marqueurs candidats sont comparés d'un laboratoire à un autre et surtout lors de workshops périodiques rassemblant les « cartographes » du génome.

En 1989, les chercheurs qui sont engagés dans la chasse aux gènes de prédisposition au cancer du sein constituent leur propre consortium international, sur l'initiative d'un chercheur français localisé au Centre international de recherche sur le cancer (CIRC). La première tâche du consortium international est de confirmer la localisation du BRCAI telle qu'elle a été proposée par King. Le consortium organise la circulation de certains outils et résultats : on sélectionne un pool de famille de références, on choisit des marqueurs de localisation chromosomique que tous les participants doivent utiliser sur leurs familles. En revanche, l'ADN des familles collectées par chaque groupe ne circule pas.

La dynamique de coopération coexiste avec une forte compétition, à la fois académique (priorité de publication) et industrielle (priorité du dépôt de brevet). Souvent les laboratoires gardent dans leurs murs les mar- 
queurs et matériaux qui leur semblent les plus prometteurs. Les familles révélant des « recombinaisons critiques » permettant de limiter la région génétique à étudier sont particulièrement prisées et secrètes. Chacun poursuit sa propre stratégie pour identifier le gène. Et plus la région du gène se resserre, plus les échanges se ferment. Le consortium se fragmente ainsi en une série de sous-groupes (3). Chaque sous-groupe est approché par ou lié à un industriel. Les échanges de données stratégiques sont forts à l'intérieur de chaque sous-groupe et faibles ou absents entre les groupes. $\mathrm{Si}$ bien que les réunions du consortium se vident de leur substance, jusqu'au clonage du gène en 1994 par Myriad Genetics : "Le consortium international s'est très rapidement dissous en mini-consortia qui n'ont jamais été intégrés... Il y a bien eu plusieurs réunions mais personne ne voulait présenter ses donnés critiques. À l'époque elles concernaient la localisation de recombinants critiques » (chercheuse, Berkeley). Le même scénario se répétera en 1994-95 pour le clonage de $B R C A 2$. Dans le consortium européen, chaque participant communique ses données au laboratoire anglais qui fait l'analyse statistique tandis que les échanges horizontaux entre laboratoires sont strictement limités pour réduire le risque de « fuite ». Cette asymétrie d'accès aux données, qui renvoie à une asymétrie de compétence en bio-informatique, justifie que ce soient les chercheurs anglais qui décident de breveter le gène $B R C A 2$. La fondation d'aide à la recherche sur le cancer qui soutient leurs recherches, la Cancer Research Campaign, dépose un brevet en 1995 dans le but affiché d'empêcher Myriad Genetics de «privatiser » la séquence du gène.

L'appropriation des connaissances reflète les tensions de la coopération. Si les participants se sont accordés pour faire des publications communes sur les pools de familles réunies par le consortium, il n'existe pas d'accord sur la propriété des gènes. Le Breast Cancer Linkage Consortium apparaît comme un cercle d'échange académique assez peu régulé et néanmoins soumis à une forte compétition : "Tout ça est très peu professionnel, d'un point de vue industriel. C'est vraiment une bande de scientifiques »(Lenoir, généticien). Les échanges au sein du consortium international sont gouvernés par la proximité ou l'éloignement du marché : une fois la course aux gènes achevée et les brevets déposés, les

(3) Le laboratoire de King en Californie est allié au centre de séquençage de Collins : l'équipe du Centre international de recherche sur le cancer de Lyon lié au collectionneur Lynch ; le groupe de Myriad Genetics associé à l'université de l'Utah et à la firme pharmaceutique Eli Lilly ; le groupe de Londres de la Cancer Research Campaign en Grande-Bretagne au centre de séquençage Sanger du Medical Research Council localisé à Cambridge. 
échanges ont repris sur des données d'intérêt médical (catalogue des mutations, études épidémiologiques) : "Là, on revient dans du consortium classique, sans implication commerciale. C'est de la santé publique classique » (professeur de génétique, Lyon).

Un troisième mode de production des savoirs sur les gènes du cancer du sein coexiste avec les deux modes précédents. Celui-ci est typique de l'organisation de la recherche génomique aux États-Unis puisqu'il repose sur une start-up, Myriad Genetics, créée à la périphérie de l'Université de l'Utah. La nouveauté tient bien sûr à l'émergence d'une sphère privée de la recherche génomique, financée par les fonds du capital risque et par un gros contrat de R\&D passé avec Eli Lilly. La mobilisation de fonds privés permettait à M. Skolnick, fondateur de la start-up, de changer l'échelle de ses recherches et de s'équiper pour faire de la biologie moléculaire et du clonage positionnel (équipement en informatique et en séquenceurs automatiques). Pour autant, Myriad Genetics demeure une petite firme jusqu'au clonage de $B R C A I$ (une trentaine de personnes en 1994) et se trouve en compétition avec des centres de génomique publiques de plus grande taille comme celui des NIH dirigé par F. Collins. Outre le branchement sur le capital privé et le changement d'échelle de la recherche génomique, nous pensons que les avantages compétitifs de Myriad Genetics dans la chasse aux gènes reposent sur une division du travail bien rodée avec l'Université de l'Utah et, facteur plus original, sur le gisement de population qui lui sert de matériel de recherche, à savoir les familles des Mormons. Autrement dit, Myriad Genetics bénéficie d'une double proximité, celle de l'Université de l'Utah et celle de la population des Mormons. Détaillons notre propos. Skolnick est un généticien des populations et un informaticien (il menait ses travaux dans le département d'informatique médicale de l'Université de l'Utah). Après des travaux de génétique des populations dans une vallée des Alpes italiennes, il a gagné l'Utah pour exploiter les données familiales enregistrées à la Société généalogique de l'Utah sur les descendants des pionniers. Cette base de donnécs, the Utah Population Database, comprend les pedigrees de 1,25 million d'individus. Ces données familiales sont ensuite rapprochées du registre des cancers de l'Utah (environ 100000 cas documentés) pour repérer les cas de cancers familiaux. Outre la taille de l'échantillon, deux facteurs facilitent le travail des généticiens : premièrement, la taille des familles que l'on peut suivre sur 6 ou 7 générations (au lieu de 3 pour les patients rencontrés dans les consultations d'oncologie); deuxièmement, la proximité géographique des familles qui facilite la réalisation des prélèvements de sang pour faire le génotypage. L'avantage des familles des Mormons a compensé la petite taille de l'équipe au départ et son retard initial dans la chasse à $B R C A I$. En une année, grâce à deux familles de très 
grande taille comportant un grand nombre de personnes affectées, les chercheurs de l'Utah ont pu confirmer et affiner la localisation du gène et commencer les travaux de clonage du gène. Ces travaux ont impliqué conjointement l'Université de l'Utah et sa start-up selon une division du travail bien délimitée : l'Université, qui héberge The Utah Population Database, se charge des études sur les familles (sélection des bons pedigrees, prélèvements d'ADN) ; Myriad Genetics réalise les travaux de clonage positionnel (localisation du gène à partir de l'analyse des familles affectées par la maladie). Cette collaboration est formalisée dans des contrats de recherche, pour chaque nouvelle recherche de gène (les recherches en cours sur les maladies cardio-vasculaires, sur l'obésité et les maladies mentales épousent la même division du travail). Les deux parties se partagent la propriété des brevets. Myriad Genetics les exploite et l'Université reçoit des royalties.

Une quatrième et dernière forme d'organisation de la recherche sur les gènes du cancer du sein est plus spécifique à la situation française. Elle trouve son origine non dans la recherche génomique mais dans le réseau des Centres de lutte contre le cancer et dans le travail des cliniciens en charge de cette pathologie. À partir du début des années quatre-vingt-dix, ceux-ci mettent sur pied ex nihilo des consultations d'oncogénétique dont la fonction première est la recherche : «Je viens chercher des familles pour de la recherche. Il n'y a aucune prestation de service derrière 》 (clinicien-chercheur, Marseille), même si l'ouverture de telles consultations n'est pas sans susciter une demande médicale : "Il y avait des gens qui venaient de très loin pour cette consultation qui n'était que de recherche, et qui commençait à se médicaliser ». La mise en réseau est rapide, soutenue par la Fédération des centres de lutte contre le cancer: «En l'espace d'un ou deux ans, les vingt centres de lutte contre le cancer se dont dotés de consultations, soit à temps complet, soit à temps partiel ». Ce réseau de correspondants devait faciliter la collecte des familles qui, en France, sont susceptibles d'être dispersées dans plusieurs régions : "Notre idée était d'établir un réseau de travail pour avoir des correspondants un peu dans les villes principales » (professeur, Clermont-Ferrand). Les échantillons sont échangés entre les centres. La Ligue contre le cancer finance la création de petites unités hybrides qui associent consultation d'oncogénétique, recherche sur les gènes de prédisposition, laboratoire de stockage des échantillons et laboratoire d'analyse susceptible à terme, dès que les gènes auront été identifiés, de fournir des tests. Ainsi, la Ligue du département de l'Ain finance la création d'une petite équipe hospitalo-universitaire à Lyon. En 1991, trois cliniciens-chercheurs constituent "le Groupe génétique et cancer » de la Fédération des centres et participent à la chasse aux gènes $B R C A$ ( $B R C A /$ vient d'être localisé en décembre 1990). 
Comparé aux modes de production précédents, le modèle français se différencie par son caractère artisanal (chaque équipe associe un ou deux chercheurs et quelques techniciens) et sa dispersion dans les établissements clinico-hospitaliers. Ce n'est pas l'université, et encore moins l'alliance entre l'université et l'industrie comme aux États-Unis, qui impulse ces recherches, mais la clinique et une Fondation dédiée au cancer. Le cadre de production est fourni par la consultation d'oncogénétique et non par des centres publics ou privés de génomique. Cela a de fortes répercussions dans la chasse aux gènes de prédisposition dans la mesure où ces petites équipes disposent de moyens de clonage trop limités pour prétendre rivaliser avec les centres de génomique américains ou anglais.

La chasse aux gènes de prédisposition au cancer du sein épouse la tendance générale à la commercialisation et à la privatisation de la recherche médicale et plus spécifiquement la tendance à l'appropriation légale des gènes (Eisenberg, 1997). De fait, elle se termine par le dépôt de multiples brevets concurrents. Toutefois, les relations des chercheurs au marché sont très différentes.

Après qu'elle eut proposée une première localisation du gène $B R C A l$, King fut contactée par plusieurs laboratoires pharmaceutiques. Elle refusa toutefois de s'engager dans la création d'une société privée dédiée à la génétique du cancer du sein. Focalisée sur la recherche de la fonction biologique du gène, elle ne croyait pas à l'émergence d'un véritable marché des tests de susceptibilité : «Jusqu'à ce que l'on ait le gène et sache comment l'utiliser, il n'y aurait qu'un tout petit marché... J'ai dit à tout le monde qu'il fallait attendre de voir ce qu'était la biologie. Qu'on ne pouvait pas "faire un marché" sur les allèles de prédisposition " (King). De fait, elle continue à travailler à l'Université sur la fonction du gène $B R C A l$ et participe aux réflexions sur l'usage de la génétique dans le cadre de l'American Society of Human Genetics.

La position de Skolnick est toute différente : tout d'abord, l'appel au marché du capital-risque lui semble indispensable pour s'engager dans la recherche génomique à une échelle industrielle ; ensuite, l'appropriation privative de la recherche, via les brevets sur les gènes identifiés, lui apparait comme la voie la plus efficace entre la science et le bien-être médical (les brevets incitant les investisseurs à développer des innovations biomédicales utiles pour la société) ; enfin, le développement de tests de diagnostic offre des perspectives de retours financiers rapides en même temps qu'il pose les premiers jalons du marché de la médecine prédictive.

La position des cliniciens-chercheurs français est encore autre et elle a sensiblement évolué au cours des années quatre-vingt-dix : leur action de recherche se déroule dans le cadre de la clinique, sur un mode artisanal. La circulation de leurs travaux, scientifiques et techniques, se fait 
dans des espaces non-marchands : les réseaux académiques, d'une part, les transferts laboratoires de recherche-laboratoire clinique, d'autre part. Ils n'ont pas de culture de la propriété industrielle et les seules relations qu'ils aient avec l'industrie sont des échanges de services (ils proposent de tester les nouvelles techniques des firmes et ils récupèrent les résultats obtenus sur leurs échantillons). La rencontre avec le marché est tardive et brutale : confrontés à l'extension en Europe des brevets de Myriad Genetics, ils réfléchissent aujourd'hui à la négociation des conditions d'accès à leurs collections d'ADN, à la nécessité de négocier des licences (Institut Curie), voire pour certains à la création de start-up (ClermontFerrand).

\section{Les cadres de fourniture et d'usage des tests génétiques : le marché et la clinique}

À la fin de l'année 1996, bien avant que les brevets sur $B R C A /$ et $B R C A 2$ ne soient accordés, deux sociétés de biotechnologie concurrentes, OncorMed, à l'est des États-Unis, et Myriad Genetics, dans l'ouest, ont commencé à commercialiser des tests de prédisposition au cancer du sein. Des associations de malades et des chercheurs en génétique humaine se sont vite inquiétés des usages de ces tests.

Le 28 mai 1996, le Washington Post ouvrait ses colonnes au débat. Le biologiste moléculaire W. Gilbert, très impliqué dans le lancement du programme génome humain et un temps conseiller de Myriad Genetics, y défendait l'utilité du test pour les femmes à « haut risque » qui pourront contrôler les facteurs environnementaux augmentant encore leur risque, bénéficier de mammographies plus fréquentes, éventuellement choisir une chirurgie préventive. A l'inverse, la biologiste de Harvard, R. Hubbard, membre du Council for Responsible Genetics, critiquait le hiatus entre le développement des pratiques de dépistage marchand et la faiblesse de leur utilité médicale. Elle rappelle les controverses sur les bénéfices du dépistage mammographique chez les jeunes femmes ou sur ceux de la chimioprévention par prise de tamoxifène. Elle affirmait que les tests ne font que créer une nouvelle catégorie de femmes à risque sans contrepartie significative en termes de prise en charge.

Aux États-Unis, on a vu apparaître une nette séparation entre la recherche clinique, qui avait jusqu'ici fourni les tests génétiques, et un marché du dépistage génétique, de la médecine prédictive, qui se met en place à côté du marché des produits et des techniques thérapeutiques. Cette séparation nourrit un débat public sur l'usage des savoirs génétiques 
qui est d'autant plus vif que les traditions d'intervention des associations de patients ou d'ex-patients y sont plus importantes. À l'inverse, en France, les tests continuent encore aujourd'hui à être fournis dans le cadre de la recherche clinique et des consultations d'oncogénétique sans grande discussion publique de leur intérêt ou de leurs usages. On peut donc opposer deux modèles de production et d'usage des tests génétiques. Aux États-Unis, un marché privé se met en place autour de deux acteurs centraux et associés, le laboratoire de diagnostic privé et l'organisation du managed care (HMOs et MCOs). En France, c'est la clinique et la profession médicale qui assurent la production des tests en routine et la régulation de leur usage médical tandis que le cadre économique et juridique de recours aux tests génétiques n'est pas encore formellement défini.

Précisons les contours du marché des tests BRCA tel qu'il se met en place aux États-Unis autour du monopole industriel et commercial de Myriad Genetics. Cette start-up a adopté d'emblée une stratégie focalisée sur la commercialisation de tests génétiques. L'accord signé en 1991 avec Eli Lilly prévoyait un partage des droits de la manière suivante : le laboratoire pharmaceutique recevait la propriété des applications thérapeutiques dérivées des gènes tandis que Myriad Genetics se réservait l'exclusivité des droits sur les usages diagnostiques. Les différents accords conclus ensuite par Myriad Genetics avec des grands laboratoires pharmaceutiques sur des pathologies variées (cancer, maladies cardio-vasculaires, obésité, asthme, dépression, etc.) reconduisent le même partage des droits et des marchés. Myriad Genetics a fondé une filiale dédiée à ce marché de la « médecine prédictive et personnelle », Myriad Genetic Laboratories.

Fait plus original, les tests BRCA ne seront pas fournis aux cliniciens sous forme de kits, mais ils seront réalisés dans une usine à tests qui est implantée à proximité immédiate des laboratoires de $R \& D$. Ce choix a été en partie dicté par la complexité des gènes et des tests BRCA : «The decision to set up the platform was driven by this biology of the gene » (Ward, directeur médical de Myriad's labs). En effet, les mutations sont réparties sur l'ensemble du gène, qui est de très grande taille (10 fois la moyenne), il n'y a pas de mutation dominante que l'on retrouve dans une majorité des cas, et on continue encore aujourd'hui à identifier et cataloguer des mutations prédisposantes. Compte tenu de l'impossibilité de mettre en boite un test simple qui aurait détecté un petit nombre de mutations que l'on aurait retrouvé dans un grand nombre de cas, Myriad Genetics s'orienta vers la réalisation de tests dans ses propres laboratoires en faisant fond sur ses savoir-faire en matière d'automation et d'informatique. Dans le courant de l'année 1996, les responsables de la firme pri- 
rent la décision de construire une plate-forme technique automatisée à même de réaliser le séquençage direct du gène.

Cet outil industriel, unique en matière de tests génétiques, est un élément majeur dans la stratégie de marché de Myriad Genetics. Le pouvoir de la firme repose de manière non négligeable sur son savoir-faire industriel, qui se renforce avec la croissance rapide du nombre de tests réalisés. La procédure de test, désormais bien établie, a été certifiée par le Département de la santé (elle satisfait aux standards de qualité du Clinical Laboratory Improvement Amendments, CLIA) (même si les limites de la technique utilisée, le séquençage direct, sont discutées par certains cliniciens-chercheurs, notamment en ce qui concerne la capacité à identifier les mutations de grande taille). Myriad Laboratories est aujourd'hui le centre de référence pour les tests BRCA aux États-Unis, sinon dans le monde.

Les brevets constituent la seconde pièce maîtresse dans la stratégie de construction du marché des tests poursuivie par Myriad Genetics. L'appropriation légale se révèle ainsi le complément indispensable à l'appropriation par les pratiques. La firme détient aujourd'hui une couverture brevet très étendue sur les deux gènes, BRCAI et BRCA2 (4). Pour autant, ce n'est qu'en 1998 que la propriété industrielle de Myriad Genetics s'est consolidée. Jusque là, deux batailles de brevets se profilaient : l'une avec la société OncorMed, à laquelle l'Office américain des brevets avait accordé le premier brevet sur le gène $B R C A I$; l'autre, avec le Cancer Research Campaign anglais qui avait déposé un brevet sur BRCA2. Ce n'est pas la justice qui a résolu les conflits d'antériorité, mais un accord commercial conclu en mai 1998 entre OncorMed et Myriad Genetics. À l'issue de cet accord, Myriad Genetics a reçu tous les droits d'OncorMed ainsi que son fonds de commerce (listes de clients, relations avec des cliniciens et centres anticancéreux). Cet accord mit également un terme au litige avec le CRC puisque OncorMed était le licencié du CRC pour l'application du brevet sur BRCA2 pour l'ensemble du monde, sauf la GrandeBretagne. Comment expliquer l'effacement de la concurrence d'OncorMed ? Principalement par la solidité des actifs scientifiques ct industriels de Myriad Genetics. OncorMed ne disposait pas d'une base scientifique et technique suffisante pour défendre ses brevets face à Myriad Genetics. Non seulement Myriad Genetics possède aujourd'hui une base brevet très étendue pour étendre son marché, mais ces brevets sont eux-mêmes très « larges ». Ils revendiquent en effet tous les produits dérivés des séquences des gènes (toute reproduction de la séquence tombe

(4) Cinq brevets aux États-Unis sur BRCAI et un brevet sur BRCA2 et des demandes d'extension en Europe en cours d'examen par l'Office européen des brevets. 
sous leur coup) et toutes les applications potentielles, diagnostiques et thérapeutiques. Ces brevets ont certes été contestés par des associations de malades fédérées dans une coalition internationale animée par la Foundation on Economic Trends de Rifkin (5). Mais, dans ce cas, comme plus généralement en matière de brevetabilité du vivant, les critiques ont peu infléchi l'évolution de la jurisprudence : les brevets de séquence reconnaissant un contrôle sur la totalité des usages industriels d'un gène sont aujourd'hui monnaie courante.

Deuxième caractéristique de la situation américaine, l'usage médical de ces tests est source de nombreuses interrogations, de la part des professionnels comme des associations de patientes (par exemple, la NBCC et la National Alliance of Breast Cancer Organization). La gestion du risque est compliquée par les incertitudes de la prédiction et par l'absence de preuve de l'efficacité des interventions de prévention (mastectomie, chimiothérapie préventive). En l'état actuel des choses, les tests positifs débouchent essentiellement sur l'intensification de la surveillance mammographique et l'inscription dans des programmes de dépistage précoce du cancer propres aux femmes à haut risque de 25 à 40 ans.

Dans ce contexte, les perspectives de mise sur le marché des tests suscitèrent de vives controverses. Les acteurs des programmes publics de génomique y étaient plutôt hostiles. En 1994, l'American Society for Human Genetics élabora des guidelines sur les tests de prédisposition au cancer du sein et de l'ovaire qui recommandaient la fourniture de ces tests dans un contexte de recherche clinique, à même de pouvoir offrir le conseil génétique et le suivi de long terme nécessaires, du moins tant que les risques de cancer associés aux différentes mutations des gènes $B R C A$ n'auraient pas été déterminés : « ... in the interim, the utilization of existing clinical geneticists to provide theses services offers the best approach for maximizing the benefit and minimizing the potential harm associated with testing ». Les recommandations du National Advisory Council for Human Genome Research vont dans le même sens : "It is premature to

(5) "L'opposition au brevet recouvre plusieurs registres de justification : tout d'abord, le droit des brevets ne peut s'appliquer à des entités qui sont déjà dans la nature (on retrouve cet argument dans l'histoire de la brevetabilité des produits chimiques et biologiques); ensuite. ces organisationss s'opposent à l'existence d'un droit exclusif d'exploitation commerciale sur des techniques d'intérêt médical qui ne seront pas accessibles aux personnes de faibles ressources (c'est l'argument de la non-brevetabilité pour impératif de santé publique) ; enfin. les opposants interviennent sur les critères de brevetabilité eux-mêmes et notamment sur la largeur des revendications des brevets Myriad » (Rifkin, 1998). 
offer DNA testing or screening for cancer predisposition outside a carefully monitored research environnement » (National Advisory Council for Human Genome Research, 1994).

En 1996, Collins, directeur du programme génome humain réitère le même avis. Il répond alors à l'offre de l'Université McGill de réaliser des tests sur une mutation qui est répandue parmi les femmes juives ashkénazes. Outre la fiabilité du test, l'Université McGill faisait valoir l'absence d'assurance privée en matière de santé au Canada et donc des conditions d'accès facilité. Collins mis en avant le cadre médical nécessaire pour dispenser ces tests. Il mit en avant les conclusions du Hereditary Susceptibility Working Group of the National Action Plan on Breast Cancer: "The current uncertainty about the appropriate medical care of mutation carriers presents a major challenge to patients and physicians ... The lack of scientific knowledge about BRCA1 and BRCA2 makes clinical uses of mutation testing premature outside of research protocols ».

De façon plus nette encore, un récent éditorial de Science s'inquiétait du manque de régulation du marché des tests génétiques qui sont commercialisés sous forme de services par plusieurs dizaines de sociétés de génomique (Holtzman, 1999), l'absence d'évaluation clinique étant susceptible de perturber le marché : «When women at risk of breast cancer learned about the predictive uncertainties of testing from sources independant of the companies offering them, they were much less eager to have tests. Thus, all stakeolders, including test developpers, will be better served if data on tests'clinical validity and utility begin to be collected before they are marketed. " L'éditorial concluait sur une nécessaire régulation des tests génétiques par la FDA, faisant ainsi écho aux propositions de la «Task Force on Genetic Testing » établie par le programme génome des NIH. Dans un rapport de 1998, celle-ci avait proposé de ne pas limiter la régulation au contrôle de qualité des laboratoires prestataires mais à instaurer un système d'évaluation de l'utilité clinique des tests en amont de la délivrance d'autorisations de mise sur le marché (Holtzman et Watson, 1998).

À l'inverse, les guidelines de l'American Society of Clinical Oncology élaborées en 1996 enregistrent l'évolution rapide de l'offre de tests génétiques. L'association des oncologues américains introduit une dissociation entre le cadre de fourniture des tests, qui peut être le marché, et la recherche clinique. Myriad Genetics inscrit ouvertement son action dans ce cadre et propose aux personnes intéressées des itinéraires de prise en charge directe accessibles par Internet. Retraçons l'itinéraire-type conçu par Myriad Genetics pour les patientes qui visitent son site. Il se décompose en cinq étapes : 
- les patientes sont invitées à remplir un questionnaire sur leur histoire familiale («Does my family history indicate an increased risk? 》);

- elles sont ensuite renvoyées dans un centre local de prise en charge du risque génétique ( Where can I find a local assessment center? along with your primary care provider, the genetic risk assessment center's experts can help you decide if genetic testing would be of value for you ») ;

- les patientes-clientes sont ensuite invitées à écouter les témoignages de cinq femmes qui ont été testées («Hear from women who have been tested ») (la cassette-vidéo des patientes dure 22 minutes);

- Myriad Genetics assiste ensuite les patientes pour la prise en charge de leur test par leur plan d'assurance («Myriad Reimbursment Assistance Programm »);

- la cinquième étape envisagc lcs options médicales possibles. Ce sont les patientes qui ont l'initiative du choix du test, ce qui rejoint les revendications de libre choix de certaines organisations féministes.

Cette médecine personnalisée et prédictive trouve un autre point d'appui dans la gestion des risques proposée par les Managed Care Organizations. Ayant à assurer au meilleur coût l'offre de soins et la prise en charge d'une grande partie de la population américaine, ces organisations sont demandeuses d'outils de gestion et de planification dont participent l'identification des facteurs de prédisposition génétiques, la quantification du risque et la différenciation des groupes de patients. Le bouclage avec les MCO est double : d'un côté, Myriad Genetics passe des accords avec des compagnies d'assurance (dont Aetna qui regroupe 23 millions d'assurés) pour solvabiliser son marché ; d'un autre côté, elle offre ses services pour une gestion intégrée du risque de santé, les MCO proposant pour leur part des options de suivi médical pour les personnes à risque. Ainsi, l'accord passé entre Myriad Genetics et Aetna US Healthcare en août 1998 prévoit que Myriad Genetics fournira des tests de susceptibilité du cancer du sein et de l'ovaire aux assurés d'Aetna qui ont un risque élevé de cancer tandis qu'Aetna fournira la couverture médicale pour les patients à risque. Il ne s'agit pas seulement de rembourser les tests, mais de pratiquer une prise en charge intégrée des personnes à risque : "BRCA analysis test information will be used by Aetna to provide improved healthcare managment for their patients ». Aetna a des contrats avec 250000 médecins et 2300 hôpitaux aux États-Unis.

Le marché des tests génétiques du cancer du sein est jusqu'ici un marché relativement modeste (la population potentiellement intéressée représente seulement $5 \%$ de l'ensemble des cas). L'offre directe de tests sur le marché, sans passer nécessairement pas le filtre de la clinique, peut augmenter légèrement ce chiffre. Pour autant, la demande de tests est en forte croissance et la part des recettes du laboratoire de génétique dans le 
total des recettes de Myriad Genetics s'accroît (en 1999, les tests représentent $20 \%$ du chiffres d'affaires de l'entreprise, à côté des revenus des contrats de recherche passés avec de grandes firmes pharmaceutiques). Les revenus des tests ont plus que doublé en 1999 (5,2 M dollars contre 2,2 en 1998). À moyen et long terme, la construction du marché des tests BRCA par Myriad Genetics à partir de 1995-1996, à savoir la création d'une plate-forme de séquençage du génome, la couverture de brevets, les accords avec les HMO's, lui sert de banc d'essai pour la mise en place du marché de la médecine prédictive à vocation universelle (Myriad Genetics commercialise déjà des tests pour les maladies cardio-vasculaires). À court terme, afin d'élargir ses recettes, Myriad Genetics a engagé une offensive en Europe pour capturer la réalisation des tests BRCA qui est assurée jusqu'ici par des laboratoires cliniques et de recherche (dans le cadre proposé par la firme, les tests pour les patients européens seraient désormais réalisés dans les laboratoires de Myriad Genetics à Salt Lake City).

Cette perspective est perçue par nombre de praticiens européens comme une menace directe à l'encontre de leur activité, tout particulièrement en France où domine un autre cadre d'usage. Les tests BRCA y sont réalisés et fournis au sein des consultations d'oncogénétiques. Les personnes ne peuvent accéder au test que par l'intermédiaire du cliniciengénéticien qui reconstitue leur histoire familiale, qui estime leur risque de prédisposition et qui, le cas échéant, leur propose de rechercher une fragilité héréditaire en réalisant un test génétique (Stoppa-Lyonnet, et al., 1997). La population susceptible d'être testée est donc étroitement définie par le conseil génétique mis en place non dans des centres de génétique médicale (comme en Grande-Bretagne) mais dans les centres anticancéreux.

Les tests BRCA sont produits directement par les cliniciens-chercheurs dans une dizaine de centres anticancéreux en France. C'est une activité hybride à la frontière de la recherche et de la clinique. Par exemple, la consultation d'oncogénétique à l'Institut Curie combine des activités de conseil génétique, de recherche des mutations sur les gènes, de dévcloppcment technologique, d'encadrement psychologique des patientes. Le clinicien-chercheur qui dirige l'équipe (4 personnes) est impliqué dans les recherches génétiques sur les analyses de liaison (il participe au Breast Cancer Linkage Consortium), dans les programmes de recherche d'épidémiologie génétique (programme BIOMED), dans des expertises collectives sur l'usage des tests, dans un groupe français d'évaluation des techniques de test utilisées par les différents laboratoires fournisseurs.

Jusqu'ici les techniques de test ont été développées directement par ces cliniciens-chercheurs, de manière assez peu coordonnée (il existe des savoirs-faire locaux qui tirent partie des ressources du laboratoire et qui ne 
sont pas facilement transposables d'un site à un autre). Le clinicien-chercheur collabore avec l'industrie ou des institutions scientifiques (l'Institut Pasteur), pour tester des prototypes ou de nouvelles techniques qui sortent sur le marché. Le cas échéant, il invente lui même quelques améliorations. La technique est appréhendée comme une ressource à acclimater et à améliorer, et non comme un produit. Les relations avec les firmes industrielles relèvent des échanges de service : «J'aurais aimé faire une collaboration avec Affymetrix pour leur faire tester en aveugle toutes les mutations $q u$ 'on a identifiées » (clinicien-chercheur, Institut Curie). Les instruments de propriété industrielle ne sont pas utilisés pour protéger les améliorations (hormis à l'Institut Gustave-Roussy où le laboratoire de test, dissocié de la consultation, a une approche industrielle). Tous ces centres de test ont évidemment de petites capacités d'analyse et ont choisi des techniques de criblage des gènes au lieu du séquençage direct développé par Myriad Genetics sur sa plate-forme de génomique. Récemment toutefois, le laboratoire de l'Institut Curie s'est équipé de robots pour faire face à l'augmentation de la demande et pour anticiper une rationalisation de l'offre de tests BRCA en France.

Cette activité de test, dispersée, n'est pas standardisée. En 1996, les onze laboratoires fournisseurs ont pris l'initiative de réunir un groupe d'évaluation et d'harmonisation des techniques de test. Ce travail collectif a commencé par la sélection d'un pool de mutations à tester. Les participants recevaient la collection complète des 35 mutants sélectionnés qu'ils testaient dans leurs murs. Les résultats étaient centralisés par un laboratoire coordinateur et discutés en réunion du groupe. Ces tests ont fait ressortir l'hétérogénéité des compétences des centres fournisseurs et ils ont produit une certaine sélection entre, d'un côté, les centres qui avaient une expérience de recherche en génétique médicale depuis la fin des années quatre-vingt et, de l'autre, les centres nouvellement équipés en séquenceurs sans expertise suffisante. Ce travail collectif n'a pas débouché sur une harmonisation des techniques de test, puisque les laboratoires pilotes ont conservé leur technique de départ (malgré quelques écarts de performance). À ce jour, l'offre de test est éparpillée dans une dizaine de centres avec des niveaux de qualité et d'utilisation des équipements très différents. D'un certain point de vue, l'offensive de Myriad Genetics pousse à une rationalisation de l'offre.

Les cliniciens-chercheurs ne produisent pas seulement les tests, mais aussi les règles d'usage médical de ces tests. Un comité ad hoc réuni entre 1996 et 1998 par l'INSERM à la demande de la Fédération des centres de lutte contre le cancer a élaboré des guidelines (INSERM, 1998). Les recommandations inscrivent la pratique des tests BRCA dans le contexte strict de la consultation d'oncogénétique. Elles portent sur la définition de 
la population de femmes auxquelles on peut proposer un test, l'organisation de la consultation et le conseil génétique, la prise en charge médicale des patientes à risque (les options de suivi médical sont particulièrement détaillées). Ces guidelines, rédigées par des praticiens de génétique moléculaire et par des experts de santé publique, fournissent un cadre de «bonnes pratiques médicales » dans le contexte de la clinique. À aucun moment, à la différence des guidelines de l'American Society of Clinical Oncology, la question du marché privé des tests n'est posée (y compris dans la liste des études souhaitées par le collectif).

Dans le contexte français, les tests sont produits dans un cadre nonmarchand. Les tests ne sont pas inscrits à la nomenclature de la Sécurité sociale et sont financés sur des crédits de recherche ou sur la partie du budget global des hôpitaux dédiée à la recherche. II s'agit donc d'un régime d'auto-production des tests par l'hôpital sur des fonds publics et sociaux. Ce régime de fourniture des tests, fondé sur des arrangements locaux au niveau des centres cliniques, est viable pour un faible volume d'activité, bien qu'en croissance. Pour autant il génère des tensions évoquées par les praticiens : tout d'abord, les tests en routine sont effectués par des laboratoires de recherche et non par des structures de transfert hospitalier (sauf à l'Institut Gustave-Roussy), ce qui peut contrarier leur activité ; ensuite, l'augmentation de la demande peut poser à terme des problèmes de couverture des coûts ; enfin, les laboratoires ne sont pas certifiés et les tests sont de qualité hétérogène, ce qui signifie que les patients n'ont pas accès au même niveau de prestation.

\section{Conclusion}

La génétique médicale du cancer du sein permet d'aborder plusieurs aspects des relations entre recherche biomédicale, médecine et marché.

Tandis que l'on met généralement en avant dans le domaine de la génomique un modèle unique de pratique de recherche, celui du chercheur-entrepreneur, nous avons pu qualifier plusieurs positions des chercheurs et cliniciens-chercheurs dans un espace science/technique/clinique/ marché, chacune ayant des définitions différentes du bien privé et du bien commun ainsi que des relations à ce qu'est la santé et le rôle de la médecine. Pour qualifier ces positions, on peut parler "d'économies » de la biomédecine au sens d'une combinaison spécifique de pratiques, de normes d'action et de jugement économique partagées par une catégorie d'acteurs. Parce que la santé joue dans les sociétés capitalistes contemporaines un rôle aussi essentiel que le pain dans les sociétés d'Ancien 
Régime, on peut même emprunter à E.P. Thompson son opposition entre une « économie politique » centrée sur les opérations des marchés et une " économie morale » incarnée dans les actions des communautés locales d'artisans et de paysans (Thompson, 1991). Les scientifiques engagés dans la génétique du cancer du sein se distribuent sur un continuum qui va du pôle de « l'économie politique » libérale (c'est la vision de Skolnick et de Myriad Genetics qui conçoivent le bien social et médical comme le résultat d'opérations marchandes, à savoir les pratiques d'appropriation par brevet, les transferts science-industrie, l'encadrement de l'itinéraire des patients par les laboratoires privés et les compagnies d'assurance) à "l'économie morale » des espaces non-marchands fondés soit sur l'organisation professionnelle (les cliniciens-chercheurs français conçoivent le bien commun en matière de santé comme l'affaire de la profession médicale qui définit les normes de bonnes pratiques et les bonnes techniques à l'écart du marché) soit sur l'alliance entre la recherche publique et les associations de malades (c'est la vision de King qui met en avant le rôle des associations de patientes, éventuellement en interaction avec des professionnels, pour faire pression afin que les tests soient mis à disposition dans des conditions équitables et offrant des garanties pour l'usage médical). Cette distribution des économies biomédicales se distingue à la fois de la vision d'une institution scientifique homogène gouvernée par des normes d'une grande originalité (les normes mertoniennes de la science) et d'une fuite en avant empreinte de néolibéralisme qui réduit les pratiques de recherche au modèle du créateur de start-up investi dans la commercialisation et à la tendance à la privatisation de la science.

La génétique du cancer du sein fait apparaître avec netteté certains des enjeux et tensions touchant aux nouveaux modes d'articulation entre science, médecine et marché. Le premier d'entre eux est le rôle majeur des brevets dans le développement de la génomique et de ses applications médicales. Les brevets de Myriad Genetics sur les gènes de prédisposition au cancer du sein sont des instruments d'appropriation des connaissances et de réservation des marchés d'autant plus puissants qu'ils sont larges et qu'ils couvrent à la fois les reproductions de la séquence et toutes les applications potentielles dérivées, diagnostiques, thérapeutiques ou à usage de screening. Leur mise en œuvre pose des problèmes de dérogation à usage de la recherche (l'Université de Pennsylvanie s'est vue interdire la réalisation de tests destinés à un consortium de recherche). Ils sont susceptibles de faire naître des monopoles qui contrarient l'accès aux soins (statement de l'American College of Medical Genetics, août 1999). Pour prévenir de telles situations, on peut songer à des formes de licences obligées et encadrées, qui assortissent le droit de propriété de règles de bonne conduite sur le marché des tests et de bonnes pratiques médicales. 
ou encore à ce que Labrusse-Riou appelle un statut de «bien commun » (Labrusse-Riou, 1988), pour qualifier des biens qui pourraient faire l'objet d'une appropriation limitée et d'une gestion finalisée pour un usage thérapeutique défini et contrôlé.

Un deuxième enjeu pour lequel la génétique du cancer du sein constitue également un banc d'essai touche à la recomposition du système de santé et à la gestion du risque génétique. Aux Etats-Unis, les deux acteurs clefs de cette transformation sont les sociétés de génomique et les organisations du managed care, qui construisent une sphère privée de la médecine prédictive. Celle-ci entretient des rapports de " négociation tendue » avec deux autres groupes d'acteurs : d'une part, avec les animateurs des grands programmes publics de génomique qui se sont efforcés de tempérer cette évolution ; d'autre part, avec les associations de femmes et de patientes comme la National Breast Cancer Coalition qui tentent de peser sur les programmes de recherche et de prévention par le lobbying et la contre-expertise. En France, l'intégration des savoirs et des savoir-faire est également très forte, mais dans le contexte de l'hôpital épaulé par la Ligue contre le cancer. Ce sont donc les cliniciens-chercheurs qui maîtrisent toute la filière, depuis la consultation ouverte aux familles jusqu'à la réalisation des tests, le jugement sur leur utilité et la production des règles de bonne conduite. L'extension européenne des brevets de Myriad Genetics provoque la collision entre ces deux modes de production et d'usage des tests génétiques et rend particulièrement aiguë la question de leur régulation.

Une meilleure maîtrise du marché suppose la délimitation de brevets plus étroits et la création de procédures de contrôle de la validité et de l'utilité clinique des tests génétiques. Ni l'intervention des usagersconsommateurs ni la régulation professionnelle sous forme de guidelines ne peuvent suffire à orienter le marché dans le sens d'une meilleure prise en compte d'enjeux de santé publique qu'il s'agit de définir collectivement et en cours de route. De fait, la Task Force for the Genetic Testing aux États-Unis propose un encadrement des tests génétiques fournis comme services par les sociétés de biotechnologie ou des laboratoires cliniques par la FDA afin d'établir la validité et l'utilité clinique de ces tests (Holtzman et Watson, 1998). Ce que suggère la comparaison des configurations américaine et française, marquée par la faiblesse de l'intervention des usagers, est qu'une telle régulation ne peut faire l'économie ni de l'intervention étatique ni de la pluralité des pouvoirs d'expertise. 


\section{RÉFÉRENCES BIBLIOGRAPHIQUES}

American Society of Clinical Oncology, 1996, Genetic testing for cancer susceptibility, Journal of Clinical Oncology, 14, 1730-1736.

American Society of Human Genetics, 1994, Statement of the american society of human genetics on genetic testing for breast cancer predisposition, American Journal of Human Genetics, 55.

American College of Medical Genetics, 1999, Position statement on gene patents and accessibility of gene testing.

Cohen D., 1993, Les gènes de l'espoir, Paris, Robert Laffont.

Collins F., 1996, Genetic screening for breast cancer, The New England Journal of Medecine, Correspondence, 334, 18, 1200-1201.

Dausset J., 1998, Clin d'xil à la vie. La grande aventure HLA, Paris, Odile Jacob.

Eisenberg R., 1997, The move towards the privatization of biomedical research, In : Barfield C.E., Smith L.R., eds, The future of biomedical research, The AEI Press.

Gaudillière J.P., 1999, Circulating mice and viruses. The Jackson Memorial Laboratory, the National Cancer Institute, and the Genetics of Breast Cancer, In : Fortune M., Mendelsohn E., eds, The pratices of human genetics, Dordrecht, Kluwer Academics Publishers.

Gibbons M., Limoges C., Nowotny H., Schwartzman S., Scott P., Trow M., 1994, The new production of knowledge, London, Sage.

Holtzman N., Watson S., 1998, Promoting safe and effective genetic testing in the United States, Final report of the task force on genetic testing, Baltimore, The Johns Hopkins University Press.

Holtzman N.A., 1999, Are genetic tests adequately regulated?, Science, 286-409. INSERM, Expertise collective, 1998, Risques héréditaires des cancers du sein et de l'ovaire. Quelle prise en charge? Paris, INSERM Éditions.

Labrusse-Riou C., 1988, Servitudes, servitudes, In : L'Homme, la nature, le droit, Paris, Christian Bourgeois, 308-384.

National Advisory Council for Human Genome Research, 1994, Statement on use of DNA testing for presymptomatic identification of cancer risk, JAMA, $271-785$.

Rifkin J., 1998, Le siècle Biotech. Le commerce des gènes, Paris, La Découverte.

Stoppa-Lyonnet D., Blandy C., Eisinger F., 1997, Cancer du sein : évaluer le risque. Les tests génétiques donnent naissance à une médecine de l'incertain, La Recherche, 294, 72-76.

Thompson E.P., 1991, Customs in common, London, Penguin Books. 


\section{ABSTRACT}

\section{Research, medicine and the market: breast cancer genetics}

Breast cancer genetics is marked by a high level of integration of biomedical science and the market. Research on breast cancer predisposition genes is performed in the context of new partnerships between university laboratories, clinical laboratories and firms. Knowledge on genes is appropriated through patents held primarily by a US start-up, Myriad Genetics. The latest fact concerns the link between intellectual property and the building of a new medical market. Myriad Genetics, strengthened by its patents and its industrial apparatus, has developed a private genetic test market that is increasingly autonomous vis-à-vis the medical world. Our article, based on a comparison between France and the United States, analyses different modes of production of research faced with market prospects. It then compares two modes of provision and use of genetic tests, and the start-up model as opposed to the clinic model. 


\section{RESUMEN}

Ciencia, medicina y mercado: la genética del cancer del seno.

El desarrollo de la genética del cancer del seno hacia el fin de los años 80 ilustra la constitución de una esfera autónoma de los tests de predisposición genética a ciertas formas de cancer que se instala en un contexto de intensificación de las relaciones entre investigación, medicina y mercado. El artículo clarifica esta dinámica a partir de una comparación entre Francia y los Estados Unidos. Para hacerlo estudia :

- la constitución de las bases de conocimientos correspondientes a estos trabajos ;

- las estrategias de apropiación desarrolladas por los investigadores, en particular el papel crucial de las patentes ;

- las relaciones entre genetistas y oncólogos, entre realización de los tests y utilización de sus resultados.

Opone un modelo «start-up » a un modelo « clínico » de emergencia de la medicina predictiva que podemos calificar respectivamente de economía « moral » y de economía « política » de los tests genéticos. 